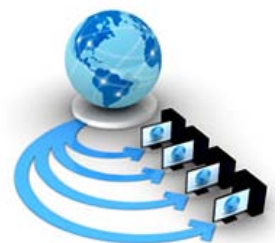

International Journal of Advanced Research in Computer Science

RESEARCH PAPER

\author{
Available Online at www.ijarcs.info
}

\title{
DETECTION OF EDGES FOR MEDICAL APPLICATION USING THEMATICAL MORPHOLOGY WITH MULTI-DIRECTIONAL STRUCTURING ELEMENTS
}

\author{
Sukhneet Kaur \\ ECE Department \\ Punjabi University, \\ Patiala,India
}

\author{
Beant Kaur \\ ECE Department \\ Punjabi University, \\ Patiala,India
}

\begin{abstract}
Edge detection is useful for identification of points in digital images. It gives the curve line where there is a change in intensity values or brightness in pixels of an image. It is very much useful in geographical areas, medical and number plate detection. In this paper, edge detection is used for medical application using mathematical morphology with multi-directional structuring elements.
\end{abstract}

Keywords: edge detection, mathematical morphology, dilation, erosion, structuring elements.

\section{INTRODUCTION}

Edge detection is used in mathematical methods to identify points in digital image. The points which brighten changes sharply are organized into set of curved line segments termed edges [1]. Edge is additionally characterized as set of connected pixels to form the boundary of objects [2]. This process decreases the amount of data by taking the derivatives of image function and filter out useless information. The motivation behind the edge detection is to extract the information about shape of object [3]. Edge detection used in image analysis, texture feature extraction, image segmentation and shape feature extraction [4].

Mathematical Morphology is the new approach for edge detection it is tool which are useful for extracting image components useful for representation and description of region shape [5]. The structuring element in mathematical morphology is used to detect the edges of image object and also for image transformation from original image into another image to find out the shape and size of object [5].

\section{RELATED WORK}

K.Y. Chan et al. (2017), introduced an edge detection Edges on the objects have to be identified for detection of interested objects from images. The proposed method was composed of modified sigmoid functions framework based an inertial measurement unit. When cameras topological structure of MSF was estimated continuously and it improves edge detection and shows lower errors. This technique was reliable for real time navigation [6].

B. Yang et al. (2017), introduced the edge guided salient object detection. For this proposed method derive two scales of nested super pixels from edge guided segmentation and then calculate a coarsesaliency ma on the fixer scale by adopting color contrast spatial prior and boundary prior. It demonstrates that the proposed saliency model consistently outperforms the state of the art saliency model [7].

J. Hao et al. (2017), proposed the edge detection with Non uniform Assembly coefficients and self-adaptive merge weights for RGB images. Edge detection with nonuniformly assembly coefficients and equal merge weight by mean of multiscale structure element for RGB image. It proposes an improved edge detection method with nonuniform assembly coefficients and self-adaptive merge weight for RGB image. This method improves the performance of edge detection [8].

C. Akinlar et al. (2017), proposed the colour edge and segment detection by edge drawing evaluate colored with different color spaces and vector gradient operates with in the precision recall frame work of the widely used barkeley segmentation dataset and Benchmark and conclude that color edge detection is in general superior to gray scale edge detection [9].

K. Lakhani et al. (2016), introduced analyzing edge detection techniques for feature extraction in dental radiographs. The proposed method implemented for set of 20 radiographs and no of pixel in each image has been calculated. Gaussian filter applied to sharpen the edges of image. It helps to detect discontinuities in dental radiographs[10].

C.I.Gonzalez et al. (2016), proposed the edge detection for optimization of interval type and fuzzy systems for image edge detection. This paper uses type 2 fuzzy logic in edge detection method is to provide them ability to handle uncertainty in processing world images. In this fuzzy logic cuckoo search (CS) and genetic algorithm (GAS) are applied[11].

X.Li et al. (2015), proposed a robust mosaicking procedure for high spatial resolution remote sensing images. Image mosaicking can resolve the problem by creating a new large area from multiple images with overlapping areas it aimed at processing HR remote sensing images of urban areas[12].

G.Hassan et al. (2015) proposed the retinal blood vessel segmentation approach based on mathematical morphology. This paper present blood vessel segmentation approach, which used in computer based retinal image analysis to extract the retinal image vessel. Mathematical morphology and $\mathrm{K}$ means clustering are used to segment the vessels it provides average accuracy of $95.10 \%$ and best accuracy of 96.25\%[13].

V.Gonzalez Castro et al. (2014) proposed the color adaptive neighborhood mathematical morphology and its application to pixel level classification. These operators have been 
applied to images in different color spaces and compared with other kinds of ASEs extended to color images and its is applied to classification of color texture images[14].

G.T.Srivakshan et al. (2012) proposed the comparison of various edge detection techniques used in image processing this paper detect a shark fish. Two operators are used are gradient and laplacian operators gradient based filters used are Roberts, Sobel, Prewitt Laplacian edge detection and canny edge detector [15].

O.Y.Hua et al. (2011) proposed a voltage sag detection based on DQ transform and mathematical morphology filter the parameters magnitude duration and phase angle jump of voltage sag accurately a method of voltage sag detection based on DQ transform and mathematical filter is present three phase is created from reference angle single phase voltage by a 60degree delay than three phase voltage is transformed into the DQ conversion of instantaneous voltage and the direct current components of DQ voltages are picked up by mathematical morphology filter compared with conventional method mathematical filter has shorter response time and higher detection accuracy[16]

C. Ducotter et al. (2004) described the scale adaptive detection and local characterization of edges based on wavelet transform the algorithm that allows to detect edges at a locally adapted scale it measures both amplitude and smoothness degree the contrast and blur level of edges points this method performed on both synthetic and real images synthetic used to investigate sensitivity to noise real images highlight the performance of images [17].

T.Chen et al. (2002) proposed a pseudo top-hat mathematical morphological approach for edge detection in dark regions. In this approach fine edge details in dark region of image were eliminated. It was useful in bright regions. This proposed algorithm was efficient for edge detail and extraction in place of shadi8ng while preserving distinguish feature [18].

\section{PROPOSED WORK}

\section{A. Traditional Operators used for edge detection}

There are various kinds of edge detection algorithm and these algorithm are differentiating based on their working principle.it has different operators have their individual different mask operator. To detect edges more easily firstly convert the colour image into gray level. Gray level image has specific range of pixels between 0 to 255 [19]. There are four operators which uses the first order derivative to detect the edges.

- Canny operators

- Sobel operators

- $\quad$ Prewitt operators

- Robert operators

\section{B. Mathematical Morphology}

Mathematical morphology with dilation and erosion has been used in the proposed technique:

\section{- Dilation}

Dilation is process to increase the maximum value in the window. After applied dilation image brightness is increased and its intensity also increases. It also extends the image and it fill the spaces using dilation[20]. This method is used to expand image objects by changing the value of 0 to 1 .

\section{- Erosion}

Erosion process is opposite to dilation process. It has minimum value. Image become more darken then original image after applying erosion[20]. It contract the image by converting pixels value from " 1 ” to " 0 ".

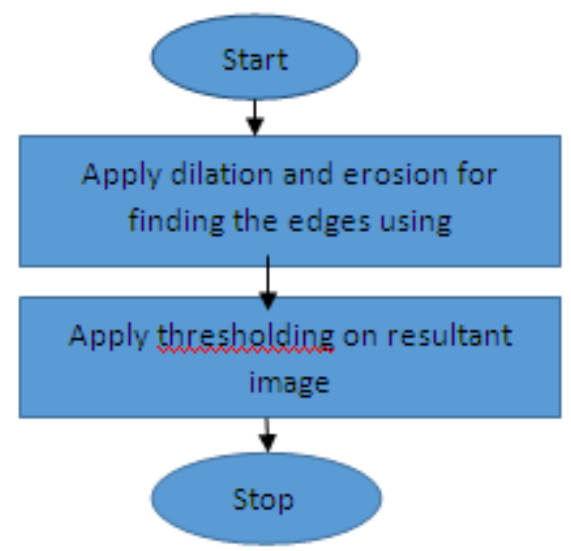

Figure 1. Flowchart for the proposed technique

- Multi- directional Structuring elements

Following multi-directional structuring elements have been used with dilation and erosion to find the edges of medical images.

$\begin{array}{cccc}\text { Se1 }= & \text { Se2 }= & \text { Se3 }= & \text { Se4= } \\ 100 & 001 & 010 & 000 \\ 010 & 010 & 010 & 1011 \\ 001 & 100 & 010 & 000\end{array}$

\begin{tabular}{|c|c|c|c|}
\hline $\mathrm{Se} 5=$ & Se6= & Se7= & Se8= \\
\hline 011 & 110 & 0001 & 1000 \\
\hline 101 & 101 & 0010 & 0100 \\
\hline \multirow[t]{2}{*}{011} & 110 & 0100 & 0010 \\
\hline & & 1000 & $\begin{array}{llll}0 & 0 & 0 & 1\end{array}$ \\
\hline $\mathrm{Se} 9=$ & Se10= & Se11= & Se12= \\
\hline 1111 & 0000 & 0000 & 0000 \\
\hline 0000 & 1111 & 1111 & 0000 \\
\hline 0000 & 0000 & 0000 & 0000 \\
\hline 0000 & 0000 & 1111 & 1111 \\
\hline
\end{tabular}

\section{Mean Square Error}

Mean square error parameter is used for finding the mathematical difference between the input and output image. The greater the value of MSE, greater will be difference between input and output image.

$$
\operatorname{MSE}=\frac{1}{M N} \sum_{y=1}^{M} \sum_{x=1}^{N}\left[I(x, y)-I^{\prime}(x, y)\right]^{2}
$$

where $I(x, y)$ is the original image, $I^{\prime}(x, y)$ is the output image. 


\section{RESULTS}

The proposed work has been implanted using MATLAB software on medical image. Followings results have been concluded using different structuring elements:

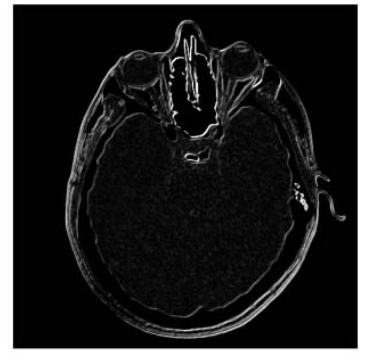

(a)

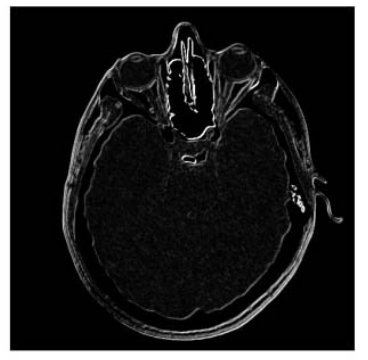

(c)

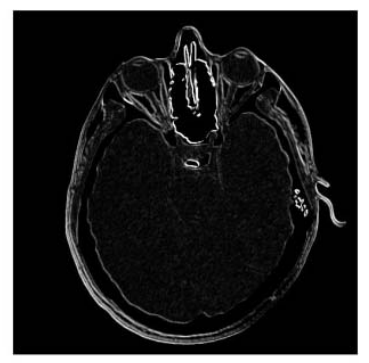

(e)

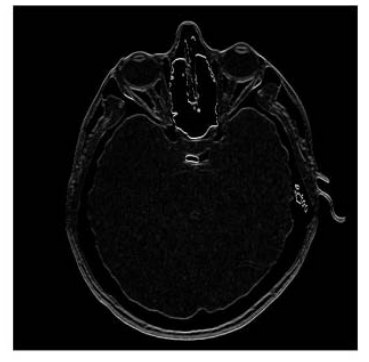

(g)

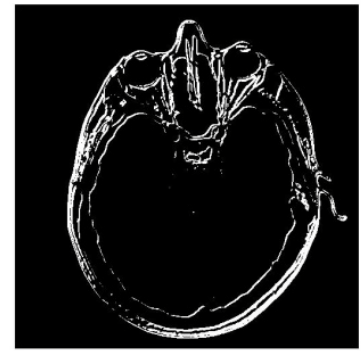

(b)

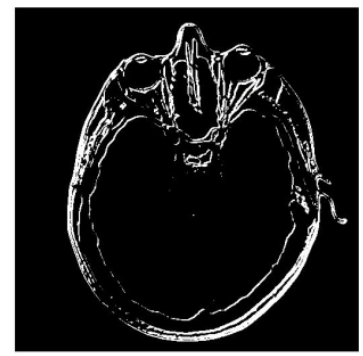

(d)

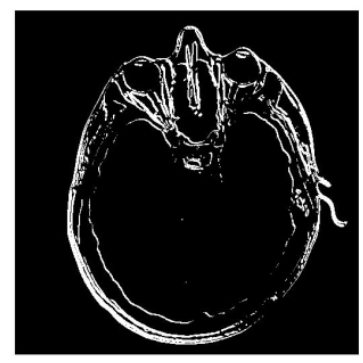

(f)

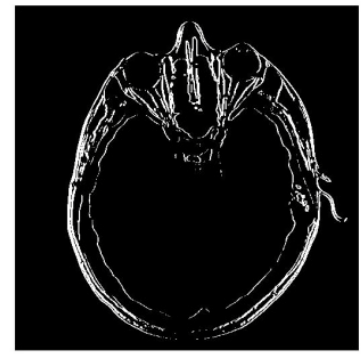

(h) 


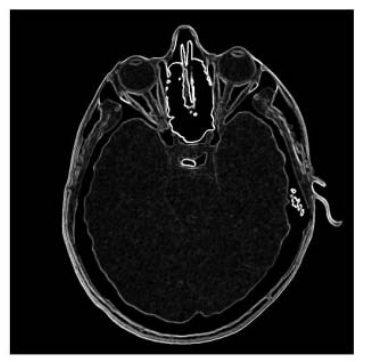

(i)

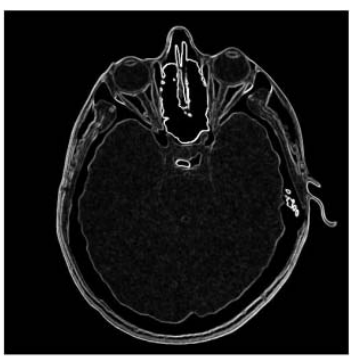

(k)

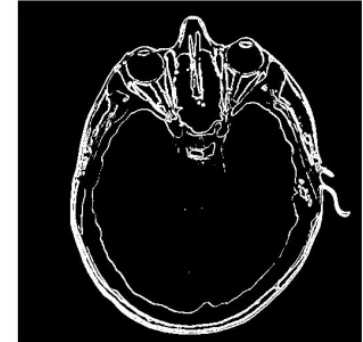

(j)

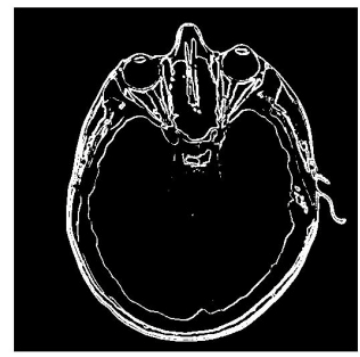

(l)

Figure 2. Results of implementation of different structuring elements a) Se1 b) Se1 with thresholoding c)Se2 d) Se2 with thresholding e) Se3 f) Se3 with thresholding g) Se4 h) Se4 with thresholding i) Se5 j) Se5 with thresholding k) Se6 l) Se6 with thresholding

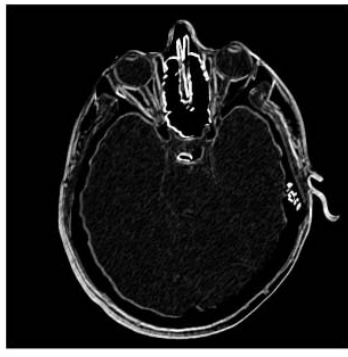

(a)

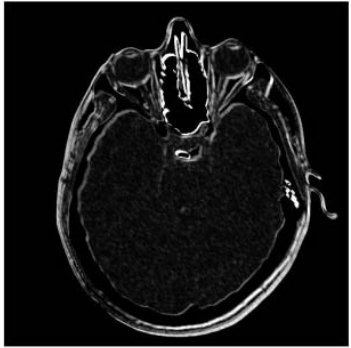

(C)

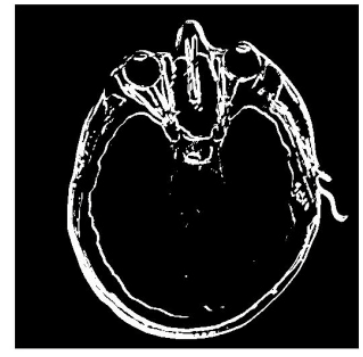

(b)

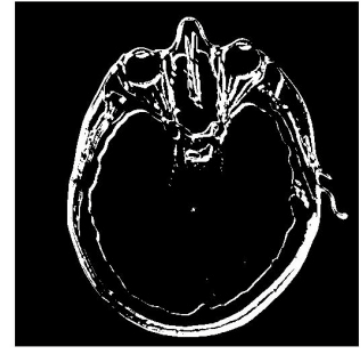

(d) 


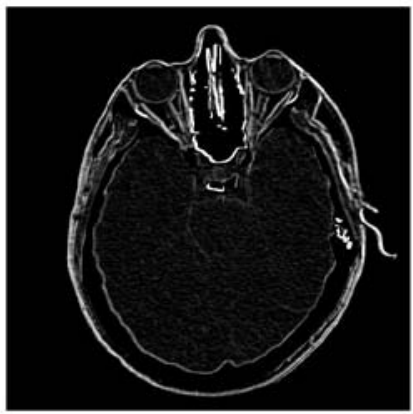

(e)

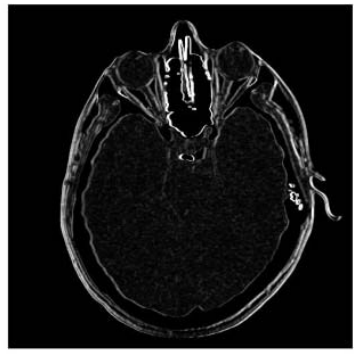

(g)

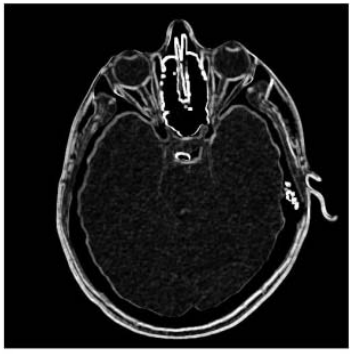

(i)

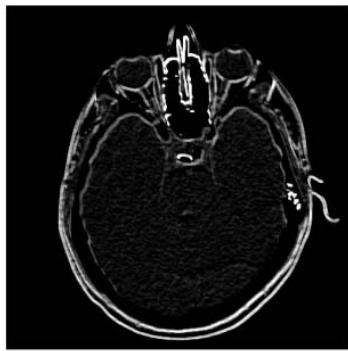

(k)

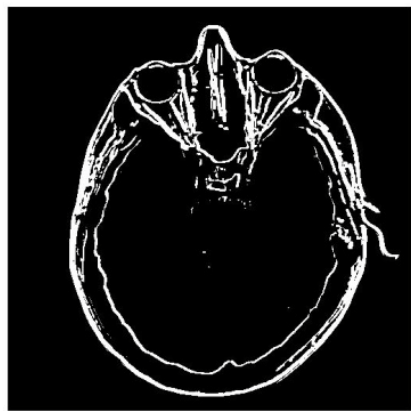

(f)

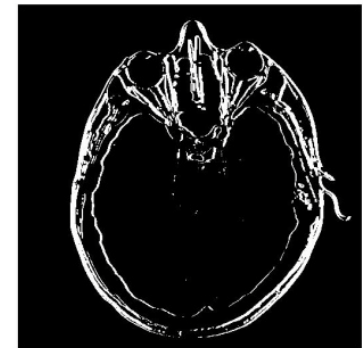

(h)

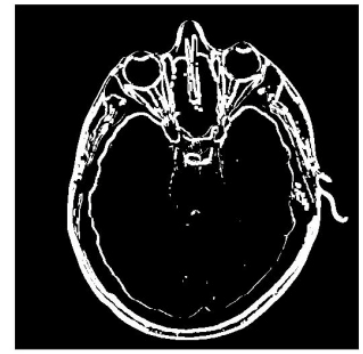

(j)

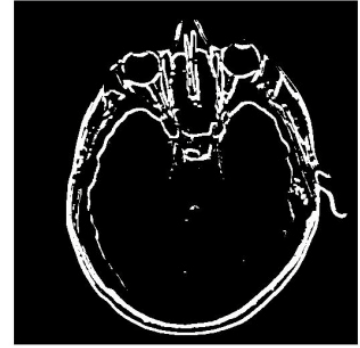

(l)

Figure 3. Results of implementation of different structuring elements a) Se7 b) Se7 with thresholding c)Se 8 d) Se8 with thresholding e) Se9 f) Se 9 with thresholding g) Se10 h) Se10 with thresholding i) Se 11 j) Se11 with thresholding k) Se12 l) Se12 with thresholding

Table 1 Mean square error using multi-directional structuring elements

\begin{tabular}{|c|c|}
\hline Structuring element & $\begin{array}{c}\text { Mean square error( at } 0.2 \\
\text { thresholding) }\end{array}$ \\
\hline Se1 & 1.5922 \\
\hline Se2 & 1.5921 \\
\hline Se3 & 1.5929 \\
\hline Se4 & 1.5925 \\
\hline
\end{tabular}

\begin{tabular}{|c|c|}
\hline Se5 & 1.5917 \\
\hline Se6 & 1.5916 \\
\hline Se7 & 1.5914 \\
\hline Se8 & 1.5915 \\
\hline Se9 & 1.5917 \\
\hline Se10 & 1.5919 \\
\hline Se11 & 1.5910 \\
\hline Se12 & 1.5916 \\
\hline
\end{tabular}




\section{CONCLUSIONS}

From the table 1, it has been observed that structuring element (Se11) has less mean square value result as compared to other structuring elements at 0.2 thresholding value. So edges can be easily detected with the help of mathematical morphology. It has been also concluded that when the matrix of structuring elements is large, the mean square value become less.

\section{REFERENCES}

[1] https://en.wikipedia.org/wiki/Edge_detection

[2] James Z. Zhang and Zijing Qin, “Edge Detection using Fast Bidemensional Empirical Mode Decomposition and Mathematical Morphology", Proceeding of the South East Conference, pp. 139-142,2010.

[3] Guang Deng and Jean-Charles Pinoli, Differentiation based edge detection using the Logarithmic image Processing Model”, Journal of Mathematical image and vision, vol.8,pp 161-180,1998

[4] Guowei Deng and FengchangXu, " Research and Analysis of Image Edge detection Algorithm based on the MATLAB", Advance in control Engineering and information Science, vol,15,pp.1313-1318,2011

[5] Beantkaur, GurdeepMohal, Palak Gupta and Jasleenkaur, “ Mathematical Morphology Edge detection for different Application: A comparative study", International Journal of Computer Science and Technology, vol2, pp.216-220,2011.

[6] Kit Yan Chan, Ulrich Engelke, NimsiriAbhayasinghe "An edge detection framework conjoining with IMU data for assisting indoor navigation of visually impaired persons" Expert systems with applications 67 (2017) 272-284.

[7] Bing Yang, Xiaoyun Zhang, Li Chen Hua Yang, ZhiyonGao“ Edge guided salient object detection” Neurocomputing 221 (2017)60-71.

[8] Jing Hao, Aili Han, Feilin Han, Lu Han” Edge detection with Non uniform Assembly Coefficients and Self Adaptive Merge Weights for RGB image” Procedia Computer Science 107(2017) 721-726.

[9] CuneytAkinlar, CihanTopal“ Color edge and segment detection by Edge Drawing(ED)J.Vis. Commun. Image R.44(2017) 82-94
[10] KanikaLakhani, BhawnaMinocha, NeerajGugnani “ Analyzing edge detection techniquesfor feature extraction in dental radiographs” Perspectives in Science (2016) 8, 395398.

[11] Claudial I, Gonzalez, Patricia Melin, Juan R.Castro, Oscar Castillo,Olivia Mendoza “ Optimization of interval type 2fuzzy systems for image edge detection” Applied Soft Computing 47(2016) 631-643.

[12] Xianghua Li, NianHui, HuanfengShen, Yunjie Fu, Liangpei Zhang “ A robust mosaicking procedure for high spatial resolution remote sensing images” ISPRS journal of Photogrammetry and Remote Sensing 109(2015) 108-125.

[13] Gehad Hassan, Naswa El- Bendary, Aboul Ella Hassanien, Ali Fahmy, Abullah M. Shoeb,and Vaclav Snasel “ Retinal blood vessel segmentation approach based on mathematical morphology” Procedia Computer Science 65(2015) 612-622.

[14] Victor Gonzalez-Castro, Johan Debayle, Jean- Charles Pinoli“ Color Adaptive Neighborhood Mathematical Morphology and its application to pixel classification" Pattern recognition letters 47(2014) 50-62.

[15] G.T. ShrivakshanDr. C. Chandrasekar“ Comparison of Various Edge Detection Techniques and in Image Processing” International Journal of Computer science Issues, Vol.9, Issues 5, No 1, September 2012

[16] Ou-yang Hua, Bu Le-ping, Yang Zhong-lin “ Voltage Sag Detection Based on Dq Transform and Mathematical Morphology Filter” Procedia Engineering 23(2011) 775-779

[17] C, Ducottet, T. Fournel, C. Barat " Scale-adaptive detection and local characterization of edges based on wavelet transform” Signal Processing 84 (2004) 2115-2137.

[18] T. Chen, Q. H. Wu, R. Rahmani- Torkaman, J. Hughes “ Pseudo top-hat mathematical morphological approach to edge detection in dark regions” Pattern Recognition 35(2002) 199210.

[19] SabanOzturk and BayramAkdemir," Comparison of Edge detection Algorithm for texture Anaysis on Glass Production”, World Conference on Technology, Innovation and Entrepreneurship, vol.195,pp.2675-2682,2015.

[20] Reecha Sharma and Beantkaur, “ Detection of Edges using Mathematical Morphology for X-ray images,” International Journal of Engineering Science, vol.5,pp230-238,2011. 